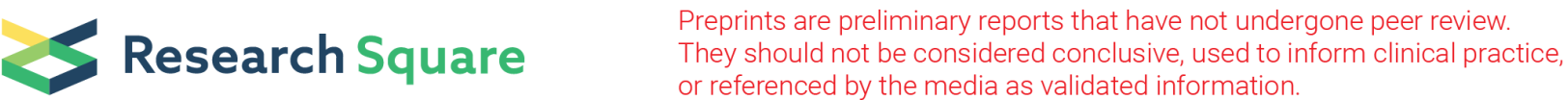

\section{MicroRNA-183-5p acts as a potential diagnostic biomarker for atherosclerosis and regulates the growth of vascular smooth muscle cell}

\section{Bin Sun}

Yidu Central Hospital

\section{Zhengkun Shan}

Yidu Central Hospital

Guoyu Sun

Yidu Central Hospital

Xiaolong Wang ( $D$ xiaolongwang_001@163.com )

Weifang People's Hospital https://orcid.org/0000-0003-3543-6659

Research article

Keywords: miR-183-5p; atherosclerosis; vascular smooth muscle cell; proliferation; migration

Posted Date: September 18th, 2019

DOl: https://doi.org/10.21203/rs.2.14613/v1

License: (c) This work is licensed under a Creative Commons Attribution 4.0 International License.

Read Full License

Version of Record: A version of this preprint was published at Journal of the Chinese Medical Association on December 31st, 2020. See the published version at

https://doi.org/10.1097/JCMA.0000000000000433. 


\section{Abstract}

Background: Atherosclerosis (AS) is a multifactorial chronic disease, and vascular smooth muscle cells (VSMCs) plays an important role in the pathology of AS. MicroRNAs regulate multiple cellular biological processes. This study aimed to investigate the clinical value of miR-183-5p in AS patients, and further explored the effects of miR-183-5p on the proliferation and migration of VSMCs.

Methods: qRT-PCR was used to test the level of miR-183-5p. The diagnostic value of miR-183-5p for AS patients was assessed by a receiver operating characteristic (ROC) analysis. Cell proliferation and migration were determined via CCK-8 and Transwell assay.

Results: MiR-183-5p was highly expressed in AS patients compared with the healthy group. Serum miR183-5p expression was positively associated with CIMT and CRP in AS patients. The ROC analysis suggested that miR-183-5p had quality to be used as a biomarker with high specificity and sensitivity for AS detection. Overexpression of miR-183-5p promoted the proliferation and migration of VSMCs.

Conclusion: MiR-183-5p is highly expressed in AS patients, and overexpression of miR-183-5p promots the cell proliferation and migration of VSMCs. MiR-183-5p may be a key molecular for the diagnosis and treatment of AS in the future.

\section{Background}

Atherosclerosis (AS), characterized by the accumulation of lipids and fibrous elements in large arteries, is the major cause of most cardiovascular events[1]. AS threatens the patients' health and even survival, also brings a heavy burden to the family and society[2]. AS is a complex process, and there are many factors that are related to the regulation of the occurrence and development of AS[3]. It is well recognized that vascular smooth muscle cells (VSMCs) play an important role in the pathological progress of AS[4]. The aberrant behaviors of VSMCs participate in the pathobiology of AS, and are related to other cellular processes such as inflammation, apoptosis and matrix alterations[5]. Therefore, regulation and stabilization of VSMCs provide a promising strategy for AS treatment[6].

MicroRNAs (miRNAs) are a type of highly conserved RNAs of 19-25 nucleotides in length, regulating the target gene expression at the transcriptional level[7]. It is known that miRNAs regulate multiple cellular biological processes, such as cell proliferation, migration, and invasion[8, 9]. A number of miRNAs have been identified to be important regulators involving in the progress of AS, suggesting the potential role of miRNA in the therapeutic strategy for $A S[2,10]$. For example, overexpression of miR-135b-5p and miR$499 a-3 p$ has been detected in the serum of AS patients, and may regulate the proliferation and migration of VSMCs via targeting myocyte enhancer factor 2C (MEF2C)[11]. MiR-365 has also been reported to be downregulated in coronary AS patients, it may regulate the pathogenesis and immune response in AS via targeting IL-6[12]. Recently, the abnormal expression of circulating miR-183-5p has been detected in both acute coronary syndrome (ACS) and myocardial infarction (MI) patients, and it is identified to be promising biomarker for the disease diagnosis, suggesting its crucial role in cardiovascular disease, 
which is related to the development of AS[13, 14]. Nevertheless, the potential role and mechanism of miR-183-5p in AS progression have not yet well investigated.

In the present study, we analyzed the level of miR-183-5p in the serum of AS patients, and investigated its clinical value. Additionally, we further explored the effects of miR-183-5p on the proliferation and migration of VSMCs.

\section{Materials And Methods}

\section{Ethnic and study participants}

This study was approved by the Ethics Committee of Yidu Central Hospital of Weifang, and each participant gave fully informed oral and written consent.

108 patients with asymptomatic AS and 72 healthy controls from Yidu Central Hospital of Weifang were enrolled in this study, all participants had no history of smoke. Patients who had a history of cardiovascular and cerebrovascular diseases, diabetes mellitus, cancer, rheumatic immune disease, acute infection disease, or on medication were excluded. The fasting venous blood was drawn for determining plasma total cholesterol, triglyceride, high-density lipoprotein cholesterol (HDL-C), low-density lipoprotein cholesterol (LDL-C) and C-reactive protein (CRP). The blood samples were isolated by centrifugation and stored at $-80^{\circ} \mathrm{C}$ for further analyses. The physical examination was performed for each participant, and the results were recorded. ATL HDI 3000 ultrasound system (Advanced Technology Laboratories, Bothell, WA, USA) was used for the measurement of Carotid intima-media thickness (CIMT).

\section{Cell culture and transfection}

Human VSMCs were purchased from the American Type Culture Collection (ATCC). Cells were grown in the Dulbecco's modified Eagle's medium (DEME; Hyclone, GE Health Care, USA) with 10\% fetal bovine serum (FBS; Gibco, Thermo Fisher Scientific, Waltham, USA). Then cells were incubated in a humidified atmosphere with $5 \% \mathrm{CO}_{2}$ at $37^{\circ} \mathrm{C}$.

MiR-183-5p mimic, miR-183-5p inhibitor, and their relative control miRNAs (mimic NC and inhibitor NC) were provided by Ribobio (Guangzhou, China). The miRNAs transfection were performed to regulate the expression level of miR-183-5p by using Lipofectamine 3000 (Invitrogen, Carlsbad, CA, USA) according to the manufacturer's instructions. $48 \mathrm{~h}$ post-transfection, cells were collected for further experiments.

\section{RNA extraction and quantitative real-time PCR (qRT-PCR)}

Total RNAs were isolated from serum or cells by using TRIzol reagent (Invitrogen, Carlsbad, CA, USA). PrimeScript RT Reagent Kit (Takara, Tokyo, Japan) was applied to reverse transcribe RNA. qRT-PCR was 
performed using SYBR green I Master Mix Kit (Invitrogen, Carlsbad, CA, USA). U6 was used as an internal control, data were expressed as fold changes relative to U6. The final expression of miR-183-5p was calculated based on $2^{-\Delta \Delta C t}$ method.

\section{Cell proliferation assay}

The cell viability was examined using CCK-8 kit (Dojindo Laboratories, Kumamoto, Japan) according to the manufacturer's instruction. The stably transfected cells were seeded in 96 -well plates at a density of $5 \times 10^{4}$ cells per well. At the indicated time points ( $\left.0 \mathrm{~h}, 24 \mathrm{~h}, 48 \mathrm{~h}, 72 \mathrm{~h}\right), 10 \mu \mathrm{l} C \mathrm{CK}-8$ solution was added to each well. The cell viability was reflected by measuring the optical density at the $450 \mathrm{~nm}$ using a microplate reader (ELx800, Bio-Tek Instruments, Winooski, VT, USA).

\section{Cell migration assay}

For cell migration assay, transwell chamber (Corning, USA) was used. Cells $\left(2 \times 10^{4}\right.$ cells/well) were seeded in chambers and cultured in serum-free DMEM, while 10\% FBS was given to the lower chamber as the attractant. Following $48 \mathrm{~h}$ of incubation at $37^{\circ} \mathrm{C}$, the migrated cells on the lower chamber were stained with $0.1 \%$ crystal violet at room temperature for $20 \mathrm{~min}$. Then the number of the migrated cells was counted under an inverted microscope (Olympus Corporation, Tokyo, Japan) using five random fields of view.

\section{Statistical analysis}

Statistical analyses were performed using SPSS 21.0 software (SPSS Inc., Chicago, IL) and GraphPad Prism 7.0 software (GraphPad Software, Inc., USA). Data were expressed as the mean \pm standard deviation. Comparisons between two groups were performed using student's t-test, and comparisons between multiple groups were analyzed by one-way ANOVA analysis with a Tukey's post-hoc test. Correlations between continuous variables were assessed using the Spearman correlation coefficient. The diagnostic value of miR-183-5p for AS patients was assessed by a receiver operating characteristic (ROC) analysis. $P<0.05$ was considered to indicate a statistically significant difference. Each experiment was performed in triplicate.

\section{Results}

\section{Clinical characteristics}

The clinical characteristics of the healthy and AS groups were recorded in Table 1. The healthy and AS groups were age and gender matched. There were no significant differences between the two groups in 
BMI, total cholesterol, triglyceride, HDL-C, LDL-C, heart rate and blood pressure (all $P>0.05$ ). However, compared with the healthy groups, the AS group had a significantly higher level in $\operatorname{CRP}(P<0.05)$.

\section{Expression levels of miR-183-5p in AS patients}

The serum expression levels of miR-183-5p were measured in both AS patients and healthy controls using qRT-PCR. It was suggested that miR-183-5p was highly expressed in AS patients compared with the healthy group $(P<0.001)$, revealing the potential role of miR-183-5p in the development of AS.

\section{The correlation of miR-183-5p with CIMT and CRP}

Considering the crucial role of CIMT and CRP for the prediction of AS, the correlations of serum miR183-5p with CIMT and CRP in AS patients were assessed. The results suggested that serum miR-183$5 p$ expression was positively associated with CIMT $(r=0.590, P<0.001$, Figure $2 A)$ and CRP $(r=0.671, P$ $<0.001$, Figure 2B)..

\section{Diagnostic value of miR-183-5p in AS patients}

A ROC curve was conducted based on the serum miR-183-5p levels to analyze its diagnostic significance for AS. As shown in Figure 3, the AUC curve was 0.907 , with the sensitivity of $82.4 \%$ and the specificity of $86.1 \%$ at the cutoff value of 2.10 . This suggested miR-183-5p to be a promising biomarker for the identification of AS.

\section{MiR-183-5p promoted cell proliferation and migration of VSMCs}

The in vitro function of miR-183-5p in VSMCs was also explored using CCK-8 assay. Firstly, the VSMCs were transfected with miR-183-5p mimic or inhibitor to regulate the gene expression in vitro. It was found that miR-183-5p mimic transfection significantly increased the miR-183-5p expression, while transfection with miR-183-5p inhibitor significantly suppressed its expression level (all $P<0.001$, Figure 4A).. Subsequently, CCK-8 and transwell assays were performed to explore whether miR-183-5p was involved in the regulation of proliferation and migration of VSMCs. It was observed that compared with the control group, overexpression of miR-183-5p significantly promoted the viability and migration of VSMCs, while downregulation of miR-183-5p exhibited the opposite effect (all $P<0.05$, Figure $4 B-C)$..

\section{Discussion}

AS is a chronic inflammatory and metabolic disease, and is the main causes of cardiovascular diseases[15]. Although great progress has been made in understanding AS development, deaths from AS 
is still high. There is still an urgent need to provide therapeutic targets for AS. In the present study, we found that miR-183-5p was highly expressed in AS patients and served as a potential biomarker for AS diagnosis, and overexpression of miR-183-5p regulated the cell proliferation and migration of VSMCs.

During the last few years, specific miRNA signatures have been illustrated in numerous studies, suggesting that miRNAs can be used as diagnostic markers for various human diseases, including AS[16, 17]. The abnormal expression of miR $-183-5 p$ has been widely reported in several human diseases. Cheng et al. suggest that miR-183-5p expression level is extremely increased in breast cancer tissues, miR-183-5p exerts oncomiRs effects in breast cancer[18]. In ACS, plasma miR-183-5p is suggested to be upregulated via small RNA sequencing (sRNA-seq), which is further validated in ACS patients[13]. Another study reveals that serum miR-183-5p level is differentially expressed in patients with type 2 diabetes mellitus (T2DM)[19]. In the present study, serum miR-183-5p was determined to be significantly overexpressed in AS patients. Clinically, our study pointed out that serum miR-183-5p was positively associated with CIMT and CRP levels in AS patients. CIMT and CRP have been widely reported to be an important biomarker for AS, and a strong predictor of future cerebral and cardiovascular events[20,21]. All evidence suggests the crucial role of miR-183-5p in the pathogenesis of AS.

As a result of the high stability and easy accessibility of miRNAs, the aberrant expression of serum miRNAs has been widely reported to be biomarker for the clinical diagnosis and prognosis of different human diseases. In AS, several miRNAs have been identified to be correlated with the diagnosis of AS. For example, Huang et al. have suggested that circulating miR-29b level is positively associated with CIMT, and may exert potential biomarker for the identification of AS[17]. Another study also suggests that miR-126 and miR-143 have certain ability to differentiate AS patients from healthy controls, and their levels are related to the severity of cerebral AS[22]. Considering the close association of miR-183-5p with plasma levels of CIMT and CRP, we performed the ROC analysis to assess the ability of miR-183$5 p$ to discriminate AS patients from healthy controls. The results suggested that miR-183-5p had the quality to be used as a biomarker with high specificity and sensitivity for AS detection.

It is well known that aberrant proliferation and migration of VSMCs play an important role in the pathogenesis of AS[5]. Evidence indicates that miR-183-5p has a regulatory effect on cellular behaviors, and further participates in the pathogenesis of human diseases. In non-small cell lung cancer (NSCLC), miR-183-5p serves as an oncogene through promoting tumor cell growth and metastasis[23]. Another study reveals that miR-183-5p exerts a potential pancreatic cancer biomarker, and overexpression of miR-183-5p promoted cell proliferation, migration, and invasion[24]. Additionally, the promoting role of $\mathrm{miR}-183-5 p$ in tumor cell progress has also been determined in several other cancers, such as breast cancer and prostate cancer[18,25]. In the present study, we further explored the regulatory role of miR$183-5 p$ in the growth of VSMCs. As expected, the current study suggested that overexpression of miR$183-5 p$ promoted the proliferation and migration of VSMCs, which suggests that miR-183-5p may promote the development of AS. Consistently, Xiao et al. have proved that in in high phosphorus induced calcification of VSMCs, the expression level miR-183-5p is increased in time-dependent manners[26], 
further demonstrating the potential role of miR-183-5p in the pathogenesis of AS. Further researches are needed to investigate the exact mechanism of miR-183-5p involved in AS.

\title{
Conclusion
}

Taken together, the results of the current study demonstrated that miR-183-5p was highly expressed in AS patients, and had the ability to be used as a biomarker for AS detection. Overexpression of miR-183$5 p$ promoted the cell proliferation and migration of VSMCs. The present study advances our knowledge into understanding the molecular mechanisms of AS involving the abnormal biological behavior of VSMCs. MiR-183-5p may be a key molecular for the diagnosis and treatment of AS in the future.

\section{List Of Abbreviations}

AS Atherosclerosis

\section{VSMCs vascular smooth muscle cells}

ROC receiver operating characteristic

\author{
miRNAs microRNAs
}

MEF2C myocyte enhancer factor 2C

ACS acute coronary syndrome

MI myocardial infarction

HDL-C high-density lipoprotein cholesterol

LDL-C low-density lipoprotein cholesterol

CRP C-reactive protein

CIMT Carotid intima-media thickness 


\section{ATCC American Type Culture Collection}

\section{DEME Dulbecco's modified Eagle's medium}

FBS fetal bovine serum

qRT-PCR quantitative real-time PCR

ROC receiver operating characteristic

sRNA-seq small RNA sequencing

T2DM type 2 diabetes mellitus

NSCLC non-small cell lung cancer

\section{Declarations}

Ethics approval and consent to participate

This study was approved by the Ethics Committee of Yidu Central Hospital of Weifang, and each participant gave fully informed oral and written consent.

\section{Consent for publication}

Consent for publication was obtained from each participant.

\section{Availability of data and materials}

All data generated or analysed during this study are included in this published article.

\section{Competing interests}

The authors declare that they have no competing interests.

Funding 
Not applicable.

\section{Authors' contributions}

BS conceived and the study, analyzed the data, and wrote the manuscript. ZS and GS conducted the experiments. XW analyzed the data and revised the manuscript. All authors read and approved the final version.

\section{Acknowledgements}

Not applicable.

\section{References}

1.Qi $M, X i n S$. FGF signaling contributes to atherosclerosis by enhancing the inflammatory response in vascular smooth muscle cells. Mol Med Rep. 2019; 20(1):162-70.

2.Liu Y, Chen Y, Tan L, Zhao H, Xiao N. Linc00299/miR-490-3p/AURKA axis regulates cell growth and migration in atherosclerosis. Heart Vessels. 2019; 34(8):1370-80.

3.Song K, Li L, Sun G, Wei Y. MicroRNA-381 regulates the occurrence and immune responses of coronary atherosclerosis via cyclooxygenase-2. Exp Ther Med. 2018; 15(5):4557-63.

4.Chen J, Cui L, Yuan J, Zhang Y, Sang H. Circular RNA WDR77 target FGF-2 to regulate vascular smooth muscle cells proliferation and migration by sponging miR-124. Biochem Biophys Res Commun. 2017; 494(1-2):126-32.

5.Zheng J, Chen K, Wang H, Chen Z, Xi Y, Yin H et al. SIRT7 Regulates the Vascular Smooth Muscle Cells Proliferation and Migration via Wnt/beta-Catenin Signaling Pathway. Biomed Res Int. 2018; 2018:4769596.

6.Hou WZ, Chen XL, Wu W, Hang CH. MicroRNA-370-3p inhibits human vascular smooth muscle cell proliferation via targeting KDR/AKT signaling pathway in cerebral aneurysm. Eur Rev Med Pharmacol Sci. 2017; 21(5):1080-7.

7.Peng P, Chen T, Wang Q, Zhang Y, Zheng F, Huang $S$ et al. Decreased miR-218-5p Levels as a Serum Biomarker in Bone Metastasis of Prostate Cancer. Oncol Res Treat. 2019; 42(4):165-85.

8.Song T, Ma K, Zhao C, Yang J, Liu J. MicroRNA-2053 overexpression inhibits the development and progression of hepatocellular carcinoma. Oncol Lett. 2019; 18(2):2043-9.

9. Huang T, Wang G, Yang L, Peng B, Wen Y, Ding G et al. MiR-186 inhibits proliferation, migration, and invasion of non-small cell lung cancer cells by downregulating Yin Yang 1. Cancer Biomark. 2017; 
21(1):221-8.

10.Su G, Sun G, Liu H, Shu L, Liang Z. Downregulation of miR-34a promotes endothelial cell growth and suppresses apoptosis in atherosclerosis by regulating Bcl-2. Heart Vessels. 2018; 33(10):1185-94.

11.Xu Z, Han Y, Liu J, Jiang F, Hu H, Wang Y et al. MiR-135b-5p and MiR-499a-3p Promote Cell Proliferation and Migration in Atherosclerosis by Directly Targeting MEF2C. Sci Rep. 2015; 5:12276.

12.Lin $B$, Feng DG, Wang F, Wang JX, Xu CG, Zhao H et al. MiR-365 participates in coronary atherosclerosis through regulating IL-6. Eur Rev Med Pharmacol Sci. 2016; 20(24):5186-92.

13.Tong KL, Mahmood Zuhdi AS, Wan Ahmad WA, Vanhoutte PM, de Magalhaes JP, Mustafa MR et al. Circulating MicroRNAs in Young Patients with Acute Coronary Syndrome. Int J Mol Sci. 2018; 19(5).

14.Zhao X, Jia Y, Chen H, Yao H, Guo W. Plasma-derived exosomal miR-183 associates with protein kinase activity and may serve as a novel predictive biomarker of myocardial ischemic injury. Exp Ther Med. 2019; 18(1):179-87.

15.Cornelissen A, Guo L, Sakamoto A, Virmani R, Finn AV. New insights into the role of iron in inflammation and atherosclerosis. EBioMedicine. 2019.

16.Saucier D, Wajnberg G, Roy J, Beauregard AP, Chacko S, Crapoulet N et al. Identification of a circulating miRNA signature in extracellular vesicles collected from amyotrophic lateral sclerosis patients. Brain Res. $2019 ; 1708: 100-8$.

17.Huang YQ, Li J, Chen JY, Zhou YL, Cai AP, Huang C et al. The Association of Circulating MiR-29b and Interleukin-6 with Subclinical Atherosclerosis. Cell Physiol Biochem. 2017; 44(4):1537-44.

18.Cheng $Y$, Xiang G, Meng Y, Dong R. MiRNA-183-5p promotes cell proliferation and inhibits apoptosis in human breast cancer by targeting the PDCD4. Reprod Biol. 2016; 16(3):225-33.

19.Meerson A, Najjar A, Saad E, Sbeit W, Barhoum M, Assy N. Sex Differences in Plasma MicroRNA Biomarkers of Early and Complicated Diabetes Mellitus in Israeli Arab and Jewish Patients. Noncoding RNA. 2019; 5(2).

20.Nezu T, Hosomi N, Aoki S, Matsumoto M. Carotid Intima-Media Thickness for Atherosclerosis. J Atheroscler Thromb. 2016; 23(1):18-31.

21.Ridker PM. From C-Reactive Protein to Interleukin-6 to Interleukin-1: Moving Upstream To Identify Novel Targets for Atheroprotection. Circ Res. 2016; 118(1):145-56.

22.Gao J, Yang S, Wang K, Zhong Q, Ma A, Pan X. Plasma miR-126 and miR-143 as Potential Novel Biomarkers for Cerebral Atherosclerosis. J Stroke Cerebrovasc Dis. 2019; 28(1):38-43. 
23.Wang H, Ma Z, Liu X, Zhang C, Hu Y, Ding L et al. MiR-183-5p is required for non-small cell lung cancer progression by repressing PTEN. Biomed Pharmacother. 2019; 111:1103-11.

24.Miao F, Zhu J, Chen Y, Tang N, Wang X, Li X. MicroRNA-183-5p promotes the proliferation, invasion and metastasis of human pancreatic adenocarcinoma cells. Oncol Lett. 2016; 11(1):134-40.

25.Waseem M, Ahmad MK, Serajuddin M, Bhaskar V, Sankhwar SN, Mahdi AA. MicroRNA-183-5p: A New Potential Marker for Prostate Cancer. Indian J Clin Biochem. 2019; 34(2):207-12.

26.Xiao Y, Du YY, Gao C, Kong W. [Dynamic alteration of microRNA in high phosphorus induced calcification of vascular smooth muscle cell]. Beijing Da Xue Xue Bao Yi Xue Ban. 2016; 48(5):756-65.

\section{Table 1}

Table 1 Demographic and clinical characteristics between groups

\begin{tabular}{cccc}
\hline Features & Healthy group & AS group & $P$ value \\
& $(\mathrm{n}=72)$ & $(\mathrm{n}=108)$ & \\
\hline Gender (males/females) & $39 / 33$ & $56 / 52$ & 0.761 \\
Age (years) & $51.22 \pm 5.58$ & $51.62 \pm 5.54$ & 0.638 \\
BMI (kg/m2) & $24.35 \pm 2.64$ & $24.77 \pm 2.98$ & 0.321 \\
Total cholesterol (mg/dl) & $193.92 \pm 29.97$ & $201.21 \pm 27.36$ & 0.070 \\
Triglyceride (mg/dl) & $169.56 \pm 31.38$ & $178.54 \pm 40.58$ & 0.114 \\
HDL-C (mg/dl) & $52.83 \pm 9.13$ & $51.52 \pm 7.97$ & 0.308 \\
LDL-C (mg/dl) & $129.85 \pm 16.67$ & $132.25 \pm 22.21$ & 0.409 \\
Heart rate (beats/min) & $76.79 \pm 6.85$ & $77.81 \pm 6.87$ & 0.328 \\
SBP (mm Hg) & $131.4 \pm 12.76$ & $132.41 \pm 13.93$ & 0.673 \\
DBP (mm Hg) & $80.69 \pm 7.62$ & $82.63 \pm 6.77$ & 0.083 \\
CRP (mg/l) & $6.38 \pm 1.91$ & $16.75 \pm 2.88$ & $<0.001$ \\
\hline
\end{tabular}

AS, atherosclerosis; BMI, body mass index; HDL-C, high density lipoprotein cholesterol; LDL-C, low density lipoprotein cholesterol; SBP, systolic blood pressure; DBP, diastolic blood pressure; CRP, C-reactive protein.

\section{Figures}




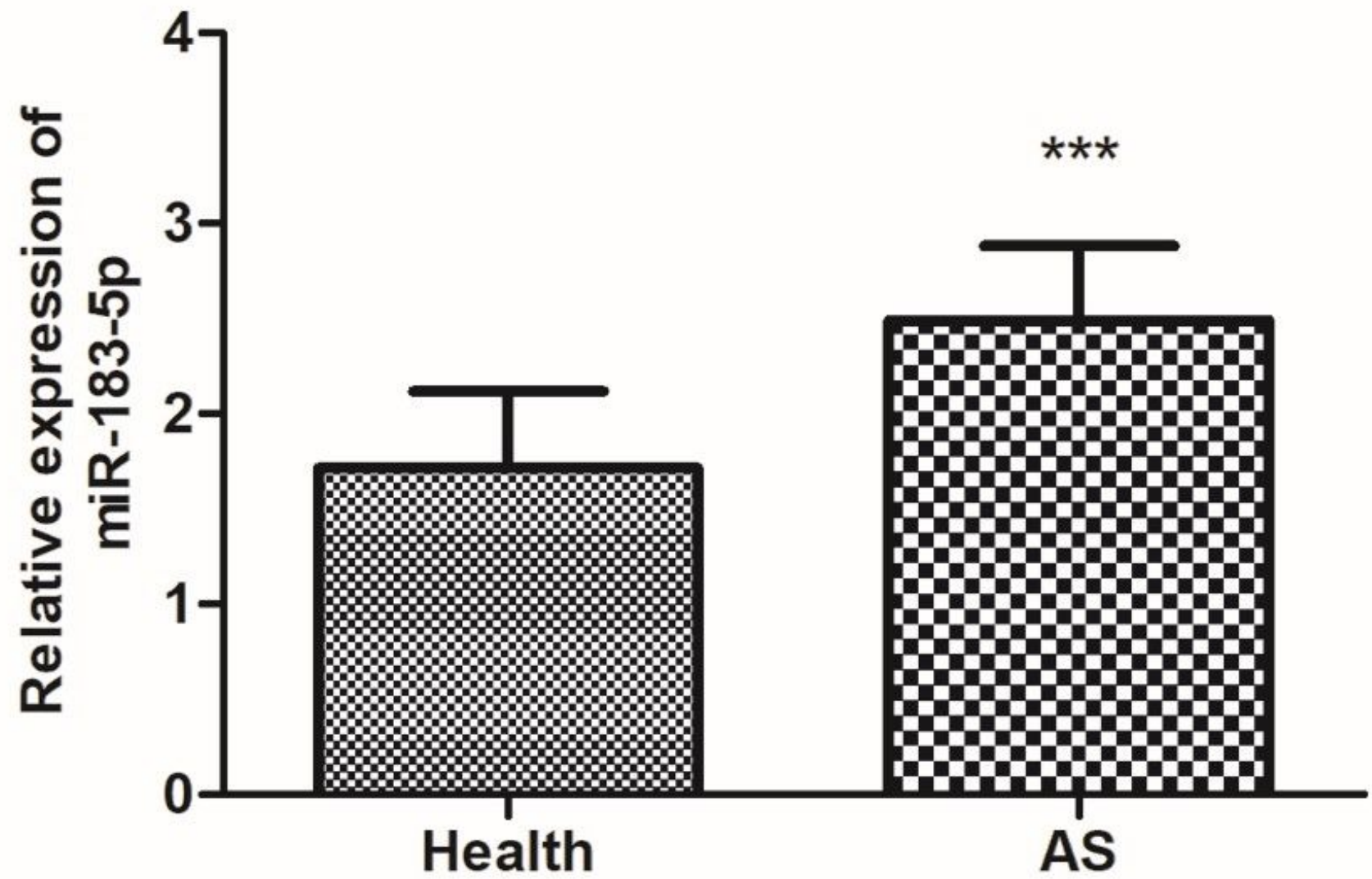

Figure 1

Serum expression level of miR-183-5p in the study population. MiR-183-5p was highly expressed in AS patients compared with the healthy group. ${ }^{* \star *} \mathrm{P}<0.001$.
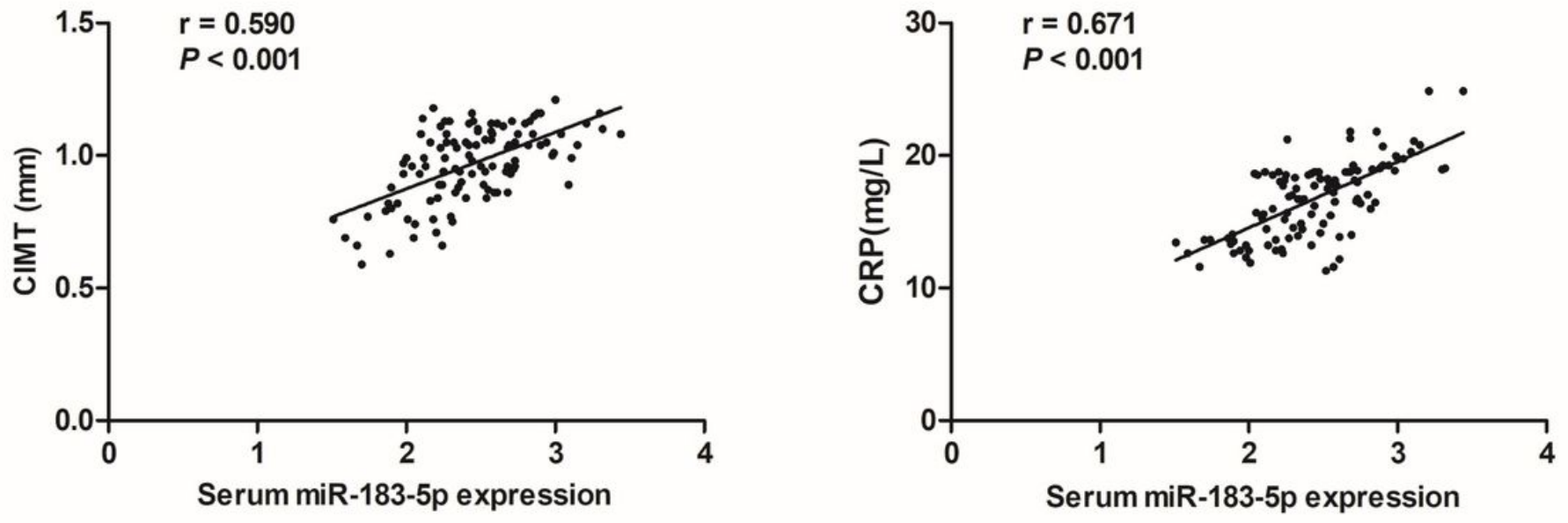

Figure 2 
Correlations of miR-183-5p with CIMT and CRP in AS patients. Serum miR-183-5p expression was positively associated with $\operatorname{CIMT}(r=0.590, P<0.001, A)$ and $\operatorname{CRP}(r=0.671, P<0.001, B)$.

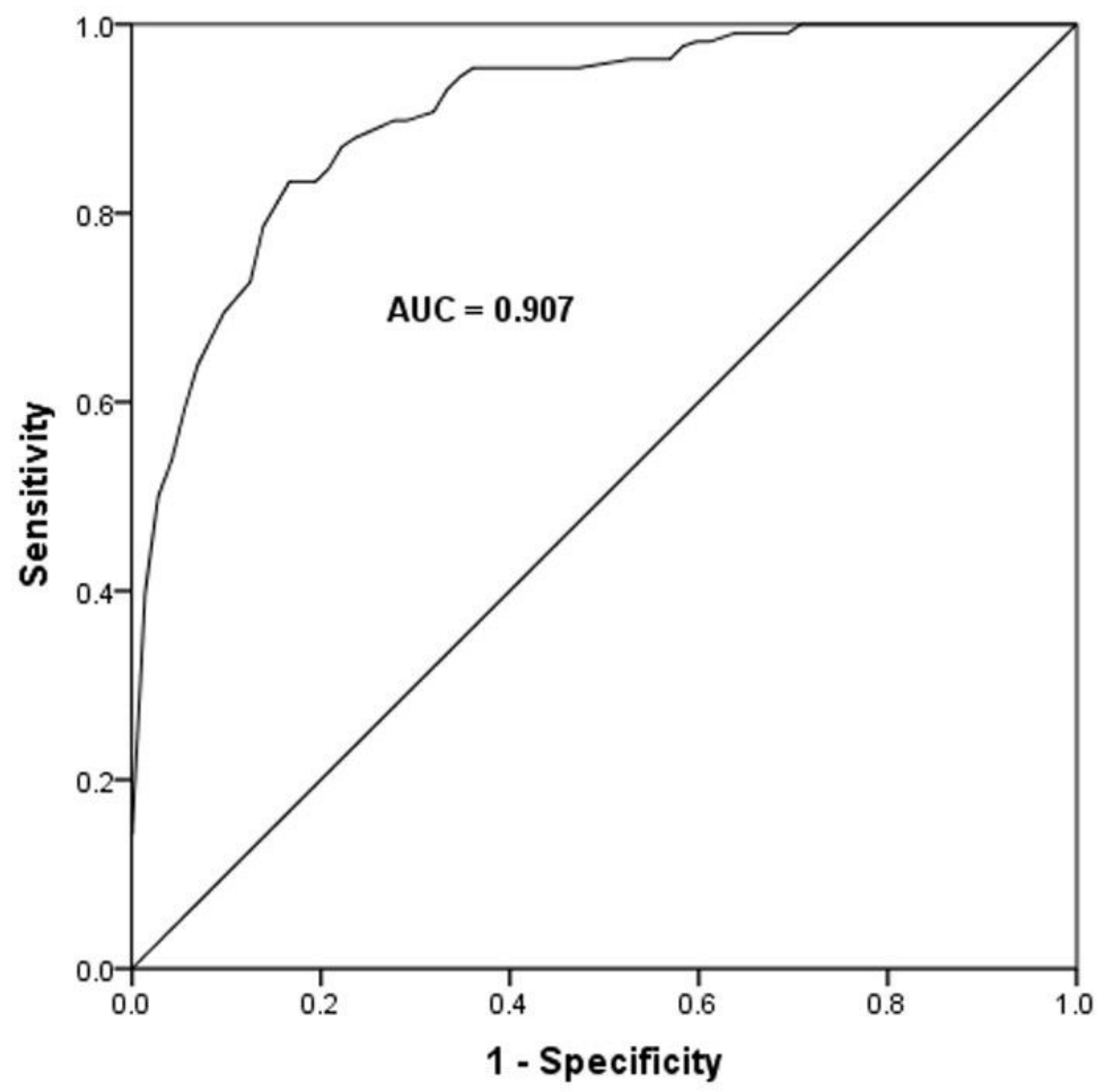

Figure 3

The diagnostic value of miR-183-5p for AS patients was assessed by a ROC analysis. Serum miR-183-5p has relative high diagnostic accuracy with an area under the curve (AUC) of 0.907. 

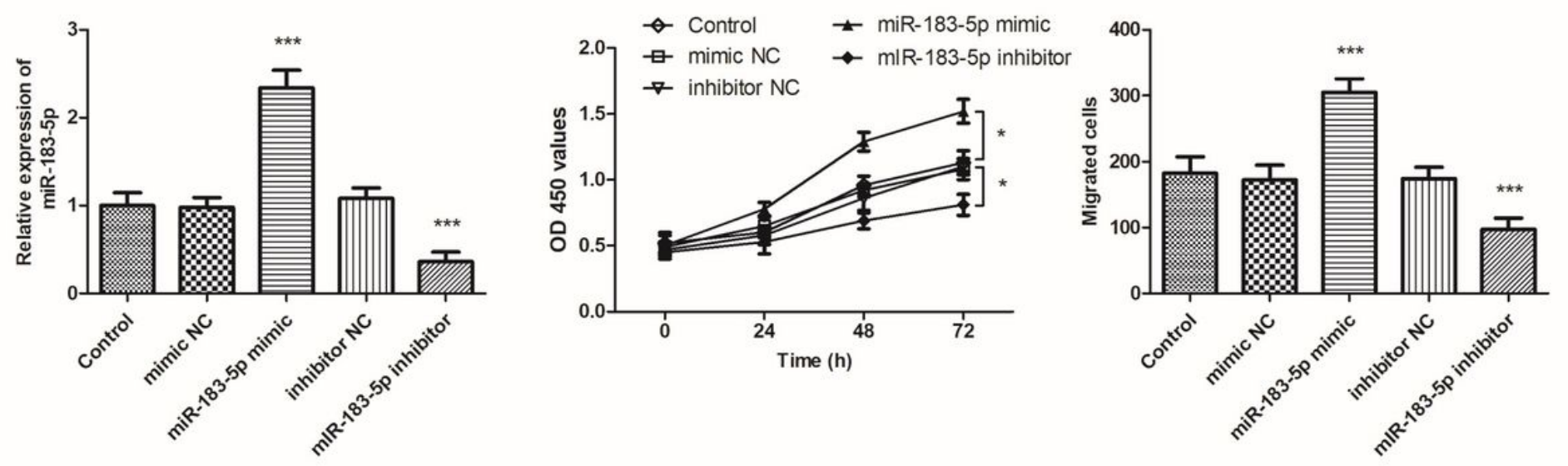

Figure 4

The effect of miR-183-5p on the biological behaviors of VSMCs. A. MiR-183-5p mimic transfection significantly increase the miR-183-5p expression, while transfection with miR-183-5p inhibitor significantly suppressed its expression level. B-C. Overexpression of miR-183-5p significantly promoted the viability and migration of VSMCs, while downregulation of miR-183-5p exhibited the opposite effect. $\star \mathrm{P}<0.05,{ }^{*} * \mathrm{P}<0.001$, compared with the control group. 